\title{
AN ERGODIC THEOREM FOR SEMIGROUPS OF CONTRACTIONS
}

\author{
S. GUTMAN AND A. PAZY
}

ABSTRACT. An ergodic theorem for semigroups of nonlinear contractions having precompact trajectories in a Banach space is proved.

1. The main result. Throughout this note $X$ will be a real Banach space, $C \subset X$ a closed subset of $X$ and $S(t), t \geqslant 0$, a semigroup of contractions on $C$, that is a family of mappings $S(t): C \rightarrow C, t \geqslant 0$, satisfying:

(i) $\lim _{t \rightarrow t_{0}} S(t) x=S\left(t_{0}\right) x$ for $t_{0} \geqslant 0, x \in C$.

(ii) $S(t+s) x=S(t) S(s) x$ for $t, s \geqslant 0, x \in C$.

(iii) $\|S(t) x-S(t) y\| \leqslant\|x-y\|$ for $t \geqslant 0, x, y \in C$.

For $x \in C$ we denote by $\alpha(x)=\{S(t) x: t \geqslant 0\}$ the trajectory starting at $x$ and by

$$
\omega(x)=\left\{y: y=\lim _{t_{n} \rightarrow \infty} S\left(t_{n}\right) x, \text { for some sequence } t_{n} \rightarrow \infty\right\},
$$

the possibly empty $\omega$-limit set of $X$. If $\omega(x) \neq \varnothing$ then it follows from its definition that $\omega(x)$ is invariant under $S(t), t \geqslant 0$, i.e. $S(t): \omega(x) \rightarrow \omega(x)$ for $t \geqslant 0$ and

$$
\lim _{t \rightarrow \infty} \operatorname{dist}(S(t) x, \omega(x))=0,
$$

where $\operatorname{dist}(z, B)$ is the distance between the point $z$ and the set $B$. Assuming, as we will do below, that for some $x \in C$ the trajectory $\alpha(x)$ is precompact, it follows easily that $\omega(x)$ is nonempty and compact. In this case, $\omega(x)$ can be given the structure of a compact commutative group and the following much stronger assertion, which is our main result, holds.

TheOREM 1 (THE ERGODIC THEOREM). Let $X, Y$ be real Banach spaces, $C \subset X$ be closed and let $S(t), t \geqslant 0$, be a semigroup of contractions on $C$. If for some $x \in C$ the trajectory $\gamma(x)$ is precompact, then $\omega(x)$ is a compact commutative group, and for every $f: C \rightarrow Y$ which is uniformly on bounded subsets of $C$ we have

$$
\lim _{T \rightarrow \infty} \frac{1}{T} \int_{0}^{T} f(S(t) x) d t=\int_{\omega(x)} f(\xi) d \xi,
$$

where $d \xi$ is the unique normalized Haar's measure on $\omega(x)$.

2. The proof of Theorem 1. Let $C \subset X$ be a closed subset of the Banach space $X$ and let $S(t), t \geqslant 0$, be a semigroup of contractions on $C$. A subset $\Omega$ of $C$ is called minimal under $S(t), t \geqslant 0$, if it is the closure of the trajectory $\gamma(y)=\{S(t) y: t \geqslant 0\}$

Received by the editors June 25, 1982.

1980 Mathematics Subject Classification. Primary 47H20, 47A35.

Key words and phrases. Semigroups of contractions, ergodic theorem, Haar's measure, $\omega$-limit set. 
for every $y \in \Omega$; it is strongly invariant under $S(t), t \geqslant 0$, if for every $t \geqslant 0, S(t)$ is a homeomorphism of $\Omega$ onto itself so that $S(t), t \geqslant 0$, can be extended as a continuous group on $\Omega$. The set $\Omega$ is equi-almost periodic under $S(t)$ if it is strongly invariant and for every $\varepsilon>0$ the set of real numbers with the property $\sup _{y \in \Omega}\|S(t) y-y\| \leqslant \varepsilon$ is relatively dense. The following proposition, whose proof can be found for example in [4, Theorem 1], is a standard result from the theory of dynamical systems.

Proposition 2. If for some $x \in C, \omega(x) \neq \varnothing$, then $\omega(x)$ is minimal and strongly invariant under $S(t)$. For each $t \in \mathbf{R}, S(t)$ is an isometry on $\omega(x)$. Moreover, if $\omega(x)$ is compact, then it is equi-almost periodic under $S(t)$.

The proof of Thereom 1 will follow easily from the next two lemmas.

Lemma 3. Let $F: C \rightarrow Y$ be uniformly continuous on bounded subsets of $C$. If the trajectory $\gamma(x)$ is bounded and $\omega(x) \neq \varnothing$ then

(3) $\lim _{T \rightarrow \infty}\left\|\frac{1}{T} \int_{0}^{T} f(S(t) x) d t-\frac{1}{T} \int_{0}^{T} f(S(t) y) d t\right\|=0$ for every $y \in \omega(x)$.

The proof of this simple lemma is omitted. It can be found, e.g., in [6, Proposition 4.1].

If $\omega(x)$ is a nonempty compact subset of $C$ there is a standard way to endow it with a commutative groups structure (see e.g. [5, Theorem 8.16, p. 394]), and hence there is a unique normalized Haar measure on it which will be denoted by $d \xi$. Moreover, if $\omega(x) \neq \varnothing$ is compact it follows from Proposition 2 that it is a minimal set consisting of almost periodic motions, and hence by [5. Theorem 9.34, p. 510] we have

Lemma 4. Let $\omega(x) \neq \varnothing$ be compact and $y \in \omega(x)$. For every continuous real valued function $h: \omega(x) \rightarrow \mathbf{R}$ we have

$$
\lim _{T \rightarrow \infty} \frac{1}{T} \int_{0}^{T} h(S(t) y) d t=\int_{\omega(x)} h(\xi) d \xi .
$$

We turn now to the proof of Theorem 1.

Proof of Theorem 1. From the precompactness of the trajectory $\gamma(x)$ it follows that it suffices to prove the theorem only for functions $f \in C(\omega(x) ; Y)$ (the space of continuous $Y$-valued functions on $\omega(x))$. Since $\omega(x)$ is compact each function $f \in C(\omega(x) ; Y)$ can be uniformly approximated by functions $g_{n}(z)$ of the form $g_{n}(z)=\sum_{k=1}^{n} h_{k}(z) e_{k}$ where $h_{k}(z): \omega(x) \rightarrow \mathbf{R}$ is continuous and $e_{k} \in Y$ for $1 \leqslant k \leqslant$ $n$. From Lemma 4 it follows readily that the theorem is true for functions $g_{n}$ of this form, and therefore by the uniform continuity of $f \in C(\omega(x) ; Y)$ and the functions $g_{n} \in C(\omega(x) ; Y)$, it is also true for any $f \in C(\omega(x) ; Y)$ and the proof is complete.

3. Concluding remarks. It is well known that if $A$ is an $m$-accretive operator in a Banach space $X$ (for the definitions and properties of such operators see e.g. [1 and 3]) then it generates a semigroup of contractions $S(t), t \geqslant 0$, on $\overline{D(A)}$ given by the exponential formula

$$
S(t) x=\lim _{n \rightarrow \infty}\left(I+\frac{t}{n} A\right)^{-n} x \quad \text { for } x \in \overline{D(A)} .
$$

For the proof of (5) see [3]. 
The main assumption of the ergodic theorem is the precompactness of the trajectory $\gamma(x)$ for some $x \in C$. This condition is clearly satisfied for all $x \in C$ if the semigroup $S(t), t \geqslant 0$, is compact for $t \geqslant 0$, i.e. for every $t>0, S(t)$ is a compact operator. A characterization of such compact semigroups, in terms of their $m$-accretive generator, is given in [2].

The compactness of the semigroup $S(t), t \geqslant 0$, is of course not necessary for the precompactness of all the trajectories of $S(t), t \geqslant 0$. It is shown in [4, Theorem 3] that if $A$ is $m$-accretive, 0 is in the range of $A$ and the everywhere defined contractions $(I+t A)^{-1}$ are compact for all $t>0$, then all the trajectories $S(t), t \geqslant 0$, are precompact and thus one can apply Theorem 1 to such semigroups.

Finally we note that Theorem 1 is an extension of a similar result in [6, Theorem 4.5] which deals with the special case where $X$ is a real Hilbert space. The conditions there assure that $\omega(x)$ lies in a finite-dimensional subspace of $X$ and it is nonempty and bounded. Hence $\omega(x)$ is clearly compact and the situation is similar to that of Theorem 1.

Acknowledgement. The authors are indebted to Professor G. R. Sell for several comments which led to an improved presentation of this note.

\section{REFERENCES}

1. V. Barbu, Nonlinear semigroups and differential equations in Banach spaces, Noordhoff, Leyden, 1976.

2. H. Brezis, New results concerning monotone operators and nonlinear semigroups, Res. Inst. Math. Sci. Kyoto Univ. No. 258 (1975), 2-27.

3. M. Crandall and T. Liggett, Generation of semi-groups of nonlinear transformations on general Banach spaces, Amer. J. Math. 93 (1971), 265-298.

4. C. Dafermos and M. Slemrod, Asymptotic behavior of nonlinear contraction semigroups, J. Funct. Anal. 13 (1973), 97-106.

5. V. V. Nemytskii and V. V. Stepanov, Qualitative theory of differential equations, Princeton Univ. Press, Princeton, N. J., 1960.

6. A. Pazy, The asymptotic behavior of semigroups of nonlinear contractions having large sets of fixed points, Proc. Roy. Soc. Edinburgh Sect. A 80 (1978), 261-271.

Institute of Mathematics, The Hebrew University of Jerusalem, 91904 Jerusalem, IsRael 